\title{
Factores determinantes de la lealtad al proveedor de servicios turísticos online ${ }^{1}$
}

Silvia Sanz Blas

Universidad de Valencia silvia.sanz@uv.es

\section{Carla Ruiz Mafé}

Universidad de Valencia carla.ruiz@uv.es

\section{Isabel Pérez Pérez}

Universidad Autónoma de Chiapas.pepisa55@hotmail.es

\section{Resumen}

El objetivo de este estudio es analizar los factores determinantes de la lealtad hacia los proveedores de servicios turísticos online a través de un modelo integrador de la influencia de la confianza en las webs turísticas con el marco conceptual de la teoría del comportamiento planificado (Theory of Planned Behaviour, TPB). El contraste de hipótesis se ha realizado a partir de una muestra de 305 compradores de billetes de avión en sitios online; para ello se utilizaron modelos de ecuaciones estructurales. Los resultados del estudio empírico indican que los constructos de dicha teoría (control percibido, norma subjetiva y actitud) ejercen una influencia significativa sobre la lealtad hacia el uso de Internet para la compra de billetes de avión. A su vez, cabe destacar la influencia positiva de la confianza en las variables del TPB. Finalmente, se plantean un conjunto de implicaciones relevantes para la gestión de empresas.

Palabras clave: compras online, webs de aerolíneas, teoría del comportamiento planificado (TPB), confianza, lealtad. 


\title{
Key drivers of loyalty towards online tourist services supplier
}

\begin{abstract}
The aim of this study is to analyze the factors determining loyalty towards online tourist services supplier with a model which integrates the influence of trust in tourism websites with the conceptual framework of the Theory of Planned Behaviour (TPB). The hypotheses were tested on a sample of 305 airline travel tickets online purchasers, with the use of structural equation models. The results of the empirical study suggest that the three variables of TPB (perceived control, subjective norm and attitude) positively influence loyalty towards Internet use to purchase airline tickets. The role of trust is especially relevant as it also boosts the effect of TPB variables. Finally, a set of managerial implications are considered.
\end{abstract}

Keywords: online shopping, airline websites, theory of planned behaviour (TPB), trust, loyalty.

\section{Introducción}

Actualmente, Internet está revolucionando la forma de operar del sector turístico al introducir importantes modificaciones en el ámbito de la comercialización de los productos y servicios turísticos, lo que permite que las empresas obtengan importantes ventajas en su forma tradicional de operar, así como en las relaciones que mantienen con sus clientes. Esta capacidad para acceder, organizar y comunicar información de forma más eficiente (Peterson et al., 1997) está transformando la forma en que las empresas se comunican e interactúan con sus clientes; así, Internet se convierte en un medio idóneo para desarrollar relaciones estables con los mismos (Vázquez et al., 2004), por lo que está transformando el intercambio transaccional en intercambio relacional, adaptándose a las necesidades de cada cliente (Gilbert et al., 1999; Zineldin, 2000).

Una de las principales características de la compra de productos/servicios turísticos por Internet es la mayor rapidez en comparar precios y la reducción del tiempo y esfuerzo dedicado a la compra. Muchos de los productos turísticos (como los billetes de avión o cualquier otro medio de transporte) presentan un alto nivel de

${ }^{1}$ Los autores agradecen el apoyo prestado por el proyecto GV/2010/069. 
estandarización; el factor precio es determinante en su elección (Kim et al., 2009). La alta competencia en precios entre los distintos operadores turísticos lleva a que cada vez sea más difícil conseguir clientes leales, por lo que resulta especialmente interesante analizar qué factores pueden contribuir a generar la fidelidad del cliente en los entornos virtuales.

Estudios previos (Lee et al., 2009; Tsai, 2006) ponen de manifiesto la influencia de las variables de la teoría del comportamiento planificado (Theory of Planned Behaviour [TPB]) (Ajzen, 1991; Taylor y Todd, 1995) en la lealtad del consumidor. De ese modo la actitud, el control percibido y la norma subjetiva se convierten en predictores significativos del comportamiento poscompra y, al mismo tiempo, son factores relevantes que motivan la decisión de uso continuado de los canales electrónicos para la compra de productos y/o servicios (Wu y Chen, 2005).

Otra variable clave en el análisis de la lealtad en los entornos virtuales es la confianza que desarrolla el consumidor hacia el establecimiento virtual (Anderson y Srinivasan, 2003; Flavián y Guinalíu, 2007), variable que intensifica el efecto que ejercen los distintos antecedentes de la lealtad en los entornos online. Así, investigaciones previas han puesto de manifiesto que la confianza es un antecedente directo y positivo de las variables del TPB (Chang, 2007; Lobb et al., 2007; Wu y Chen, 2005).

Con base en lo anterior, este trabajo tiene por objetivo analizar la influencia que ejercen las variables de la Teoría del Comportamiento Planificado (norma subjetiva, control percibido y actitud) en la lealtad hacia la compra de productos/servicios online, factores que a su vez se ven reforzados por la confianza que desarrolla el consumidor hacia el establecimiento virtual. Cabe señalar que aunque son numerosos los estudios centrados en aplicar los modelos explicativos de actitudes al uso de los servicios online, la mayoría de ellos toman como variable dependiente el estudio de las intenciones sin llegar a analizar comportamientos efectivos.

Para probar empíricamente el modelo propuesto, se han seleccionado las líneas aéreas y en concreto los billetes de avión como contexto de investigación, ya que la adopción de un enfoque relacional es una cuestión crucial en la industria aérea (Vázquez et al., 2004). Además, son los productos turísticos los más vendidos a través de Internet, demandados por más del $50 \%$ de los compradores españoles (Red.es, 2009). Por otro lado, hay que señalar que es relativamente reciente la lite- 
ratura de marketing que aborda la naturaleza de la lealtad en los entornos virtuales del sector turismo (Kim et al., 2009; Nadiri et al., 2008).

El trabajo se ha estructurado en dos partes: una primera, de contenido teórico, que comprende la revisión de la literatura, el planteamiento de las hipótesis de trabajo y la metodología de estudio; una segunda, en la que a través de un estudio empírico, utilizando una muestra de 305 internautas españoles compradores, se examina la incidencia de distintas variables en el comportamiento poscompra de productos turísticos online.

\section{Revisión de la literatura y planteamiento de hipótesis}

\section{La lealtad del consumidor hacia el establecimiento virtual}

La fidelidad o lealtad de los clientes es un constructo multidimensional que recoge, en líneas generales, cuatro aspectos esenciales: 1) que el cliente está satisfecho, 2) que recibe valor, 3) que repite compra y 4) que recomienda la empresa a terceros (Barroso y Martín, 1999). De acuerdo con Dick y Basu (1994), la lealtad del cliente es un concepto multidimensional que consiste en un comportamiento basado en una actitud; por tanto, dicha lealtad se manifiesta a través de la relación entre la actitud hacia la entidad y el comportamiento de recompra. En otras palabras, la lealtad se produce cuando la actitud relativa del consumidor hacia la entidad es favorable y, además, existe un comportamiento de compra repetido, mientras que la no lealtad consiste en una actitud relativa desfavorable combinada con la ausencia de comportamiento de recompra; por lo tanto, la lealtad no es exactamente igual a la intención de recompra, si bien parece existir un nexo de unión entre ambos conceptos como consecuencia de sus procedimientos de medición (Zeithaml et al., 1996). La aproximación exclusivamente comportamental al concepto ha sido criticada porque no diferencia entre verdadera lealtad y lealtad espuria (Dick y Basu, 1994; Shankar et al., 2003); un consumidor puede repetir constantemente la compra de un determinado bien o servicio (es fiel comportamentalmente), pero a la vez no estar actitudinalmente a favor de dicho comportamiento, por lo que estará más atento a otras alternativas en el mercado, o será más vulnerable a informaciones negativas sobre la empresa (lealtad espuria) (Shankar et al., 2003).

En este contexto, parece más completa la propuesta de Engel et al. (1982) que conciben la lealtad a la marca como la respuesta preferente, actitudinal y comportamental, hacia una o más marcas de una categoría de producto expresada por un 
consumidor en un periodo. De forma similar, Keller (1993) sugiere que la lealtad se presenta cuando las actitudes favorables hacia la marca se manifiestan en comportamiento de compra repetido.

De forma análoga, la lealtad hacia establecimientos virtuales (e-loyalty) ha sido concebida como la actitud favorable del cliente hacia una empresa, así como un comportamiento de repetición de uso/compra (Anderson y Srinivasan, 2003). Siguiendo esta línea, en este trabajo hemos concebido la lealtad al espacio web del proveedor de billetes de avión como la actitud favorable hacia el mismo, la que se manifiesta en la intención de continuar comprándole billetes de avión en el futuro.

\section{Teoría del comportamiento planificado}

La teoría del comportamiento planificado (Theory of Planned Behaviour, TPB) (Schifter y Ajzen, 1985) ha constituido la base conceptual de muchas de las investigaciones centradas en el estudio del comportamiento del consumidor (Ajzen, 1991; Taylor y Todd, 1995); en los últimos años ha sido uno de los enfoques más utilizados para explicar el comportamiento de compra. La TPB es una extensión de la teoría de la acción razonada, en la cual se incorpora la variable control percibido como antecedente de la intención/comportamiento efectivo (Ajzen, 1991) con el fin de contemplar el mayor o menor control que posee el individuo sobre su comportamiento (Taylor y Todd, 1995). De ese modo, las variables integrantes de la TPB son la actitud, la norma subjetiva y el control percibido.

La actitud es una evaluación positiva o negativa acerca del comportamiento en sí (Ajzen, 1991; Fishbein y Ajzen, 1975); hace referencia a la predisposición global hacia el desarrollo de dicha conducta; es el resultado de las creencias de los sujetos respecto al comportamiento y sus resultados, así como de la importancia que se dé a dichas creencias (Fishbein y Ajzen, 1975).

La norma subjetiva recoge la influencia de las opiniones de otras personas (familia, amigos, compañeros de trabajo) en el comportamiento del individuo (Fishbein y Ajzen, 1975; Lee y Green, 1991; Schofield, 1974). Esta variable deriva de las creencias normativas que el consumidor atribuye a los sujetos de referencia y de la motivación para comportarse según los deseos de dichas personas. 
Por último, el control percibido en el comportamiento representa las percepciones del individuo respecto de la presencia o ausencia de las habilidades, oportunidades y recursos necesarios para desarrollar la conducta (Ajzen y Madden, 1986); es el resultado de las creencias del individuo acerca de la posesión de oportunidades y recursos para llevar a cabo el comportamiento (Azjen, 1991; East, 1993; Taylor y Todd, 1995). Las percepciones del individuo respecto a su control se incorporan en el modelo como variable explicativa tanto de las intenciones como de la conducta (Ajzen, 1985), ya que la intención se convertirá en comportamiento siempre que la persona disponga de control suficiente; el resultado será mejor cuanto mayor sea dicho control (Azjen, 1991).

Las actitudes se forman según creencias del individuo sobre el comportamiento; las normas subjetivas según creencias normativas; por último, el control percibido depende del dominio que tiene el consumidor sobre las acciones que realiza y de los recursos con los que cuenta para llevar a cabo dicho comportamiento (Azjen, 1991).

Estudios previos ponen de manifiesto la influencia de las variables de la TPB en la lealtad del consumidor. Así, en el estudio de Tsai (2006) la actitud, la norma subjetiva y el control percibido son predictores significativos de la lealtad hacia los juegos online. El trabajo de Lee et al. (2009) destaca la influencia de la norma subjetiva en la lealtad hacia los servicios del móvil. Como consecuencia de la revisión de la literatura, se plantean las siguientes hipótesis tomando como marco de referencia la TPB:

$H_{I}$. La actitud influye positivamente en la lealtad hacia la página web de la venta de billetes de avión.

$\mathrm{H}_{2}$. El control percibido influye positivamente en la lealtad hacia la página web de la venta de billetes de avión.

$H_{3}$. La norma subjetiva influye positivamente en la lealtad hacia la página web de la venta de billetes de avión.

\section{Confianza}

Dimensiones de la confianza. La confianza ha sido definida desde diversas perspectivas. En el ámbito del marketing relacional hay un acuerdo bastante generalizado en concebir la confianza como la creencia de una parte (el consumidor) en la integridad y buena fe de la otra parte del intercambio (la empresa) y la consiguiente 
disposición a fiarse de ella (Morgan y Hunt, 1994). En el contexto de los entornos virtuales, Jarvenpaa et al. (2000) definen la confianza en el vendedor online como la expectativa de una de las partes acerca de los motivos y comportamientos de la otra parte.

La mayoría de las investigaciones centradas en los entornos físicos de compra considera la confianza como un constructo de carácter multidimensional formado por las dimensiones de honestidad, benevolencia y competencia (Doney y Canon, 1997; Fernández y Martín, 2006; Ganesan, 1994; San Martín, 2006).

La honestidad hace referencia a la creencia en que la otra parte asumirá sus compromisos y sus obligaciones; se cree, por tanto, en la sinceridad y el cumplimiento de las promesas del socio (Doney y Canon, 1997). La benevolencia es la creencia en que la otra parte está interesada en conseguir beneficios conjuntos y no iniciará actuaciones que pudieran dañar la relación (San Martín et al., 2004). La competencia alude a la apreciación en la empresa de unos conocimientos técnicos y una experiencia y pericia profesionales que le confieren un dominio en su campo de actividad y la ponen en condiciones de hacer bien su trabajo y ofrecer un producto o servicio con la calidad prometida (Bhattacherjee, 2002; Pavlou, 2003; Roy et al., 2001; Suh y Han, 2002). En este sentido, ofrecer al cliente un servicio rápido y sin errores o ser capaces de recomendarle el producto turístico que más se adapte a sus necesidades, son aspectos que contribuyen al éxito de la relación comercial entidad-cliente.

Confianza y actitud. La confianza interactúa con las actitudes del consumidor hacia el uso de Internet como canal de compra. Los estudios de George (2002) y Wu y Chen (2005) sugieren que la actitud es una variable mediadora de la influencia de la confianza en la intención de uso. Pavlou y Fygenson (2005) sostienen que la confianza influye de forma directa en la actitud debido a la formación de expectativas. Asimismo, McKnight et al. (1998) defienden que la confianza es una creencia que afecta a la actitud, que a su vez influye en la intención de contratar a un determinado proveedor de servicio. En el contexto de los servicios electrónicos, Suh y Han (2002) evidencian que la confianza es uno de los principales predictores de la actitud hacia el uso de servicios bancarios. De este modo, planteamos la siguiente hipótesis:

$H_{4}$. La confianza en la web de venta de billetes de avión influye positivamente en la actitud hacia el uso de Internet para la compra de billetes de avión. 
Confianza y control percibido. Investigaciones previas ponen de manifiesto la influencia de la confianza en el control percibido (Chai y Pavlou, 2002; Wu y Chen, 2005; Pavlou, 2003) sobre las transacciones online entre compradores y vendedores a través de la autoeficacia y las condiciones facilitadoras.

Siguiendo a Pavlou y Chai (2002), la confianza en el vendedor incrementa el control percibido del consumidor sobre la transacción a través de la creación de expectativas favorables sobre el comportamiento del mismo. La confianza se convierte en una condición facilitadora que incrementa la percepción del consumidor sobre los recursos de que dispone para controlar la transacción electrónica. Desde la perspectiva de la TPB, la creencia de que el vendedor virtual se comportará acorde con las expectativas del consumidor incrementa el control del mismo sobre una transacción que conlleva aparejado un elevado nivel de incertidumbre. Por consiguiente, la confianza se considera como una creencia de control que actúa como antecedente del control comportamental (Ajzen, 1985). De este modo, planteamos la siguiente hipótesis:

$H_{5}$. La confianza en la web de venta de billetes de avión influye positivamente en el control percibido del uso de Internet para la compra de billetes de avión.

Confianza y norma subjetiva. El modelo TPB descompuesto (Taylor y Todd, 1995) evidencia que la influencia de colegas y superiores es un antecedente de la norma subjetiva. Por consiguiente, si el vendedor genera confianza, los colegas, amigos y el entorno de referencia verterán opiniones positivas sobre dichos vendedores, lo que sin duda puede incrementar la motivación a comportarse conforme a las creencias normativas de esas personas (norma subjetiva) en las transacciones online (Wu y Chen, 2005).

La influencia de la confianza en la norma subjetiva ha sido contrastada en estudios previos (Chang, 2007; Wu y Chen, 2005) que ponen de manifiesto cómo la creación de confianza es un factor esencial en el proceso social de intercambio (Blau, 1964). De este modo, planteamos la siguiente hipótesis:

$H_{6}$. La confianza en la web de venta de billetes de avión influye positivamente en la norma subjetiva sobre el uso de Internet para la compra de billetes de avión.

Las hipótesis planteadas conforman el siguiente modelo (figura 1). 
Figura 1

Modelo teórico propuesto

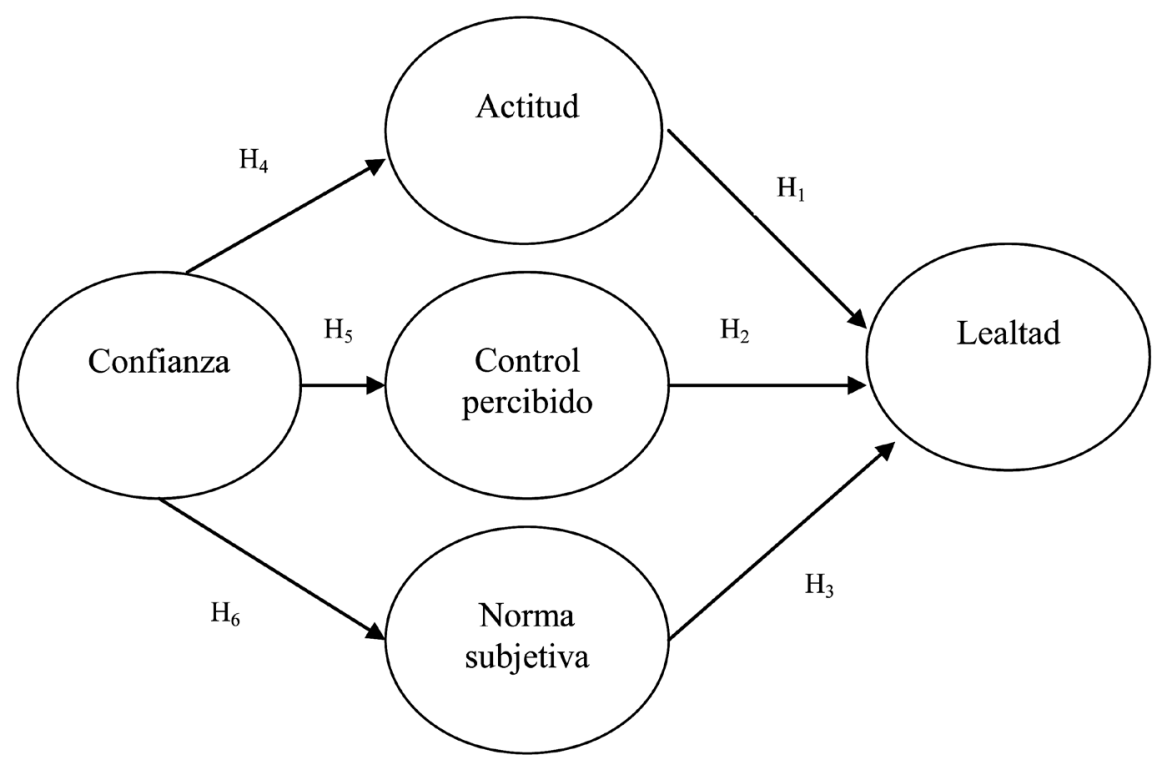

\section{Metodología}

\section{Diseño de la investigación}

Como se desprende de las hipótesis planteadas, el enfoque de la investigación es causal; las relaciones son determinadas a través de los modelos de ecuaciones estructurales. Este tipo de análisis va a permitir, por un lado, incorporar explícitamente el error de medida en los modelos de marketing y analizar su influencia en el grado de ajuste de los mismos; por otro, la evaluación simultánea de todas las variables proporcionará una información más detallada en cuanto a las interrelaciones dinámicas presentadas dentro del modelo (Hair et al., 1999; Steenkamp y Baumgartner, 2000).

Los datos objeto de análisis proceden de un estudio de mercado llevado a cabo en la comunidad valenciana durante los meses de abril y mayo de 2007. La investigación ha sido realizada utilizando una muestra de 305 internautas compradores de billetes de avión, todos mayores de 16 años. El billete de avión ha sido adquirido 
en la página web de la línea aérea o a través de intermediarios turísticos (como por ejemplo, eDreams o Carrefour). Todas las compras han sido con fines de turismo.

El método para obtener la información ha sido a través de la entrevista personal con cuestionario estructurado. El procedimiento de elección de la muestra fue por cuotas, con base en el género y edad del internauta, a partir de la caracterización de los usuarios de Internet que periódicamente realiza la Asociación para la Investigación de los Medios de Comunicación en su estudio Navegantes en la Red (AIMC, 2007).

\section{Descripción de la muestra}

El perfil de la muestra se describe en el cuadro 1. Del total de la muestra, un 67.9\% son hombres y un $32.1 \%$ mujeres. Un amplio porcentaje de los entrevistados pertenece al segmento de edad de entre 16 y 34 años (65.3\%), poseen estudios medios o superiores $(62.9 \%$ ) y un nivel de renta por encima de la media (48.9\%).

\section{Cuadro 1}

Descripción de la muestra

\begin{tabular}{|c|c|c|c|}
\hline Género & $\begin{array}{c}\% \\
(\mathrm{n}=305) \\
\end{array}$ & Ocupación & $\begin{array}{c}\% \\
(n=305) \\
\end{array}$ \\
\hline Hombre & $67.9 \%$ & \multirow{3}{*}{$\begin{array}{c}\text { Estudiante } \\
\text { Parado } \\
\text { Trabajador cuenta propia } \\
\text { Trabajador cuenta ajena }\end{array}$} & \multirow{3}{*}{$\begin{array}{c}44.6 \% \\
0.3 \% \\
8.2 \% \\
46.9 \%\end{array}$} \\
\hline Edad & $\begin{array}{c}\% \\
(n=305)\end{array}$ & & \\
\hline $\begin{array}{l}\text { Menos de } 24 \text { años } \\
\text { Entre } 25 \text { y } 34 \\
\text { Entre } 35 \text { y } 44\end{array}$ & \multirow{2}{*}{$\begin{array}{c}28.9 \% \\
36.4 \% \\
20.3 \% \\
11.1 \% \\
3.3 \%\end{array}$} & & \\
\hline $\begin{array}{l}\text { Entre } 45 \text { y } 54 \\
\text { Más de } 54 \text { años }\end{array}$ & & Educación & $\begin{array}{c}\% \\
(\mathrm{n}=\mathbf{3 0 5}) \\
\end{array}$ \\
\hline Ingresos & $\begin{array}{c}\% \\
(\mathrm{n}=305)\end{array}$ & \multirow[b]{2}{*}{$\begin{array}{l}\text { Primarios } \\
\text { Secundarios } \\
\text { Univ. Medios } \\
\text { Univ. superiores }\end{array}$} & \multirow[b]{2}{*}{$\begin{array}{l}4.8 \% \\
32.3 \% \\
24.6 \% \\
38.3 \%\end{array}$} \\
\hline $\begin{array}{c}\text { Por debajo de la media }(<900 €) \\
\text { En la media }(=900 €) \\
\text { Por encima de la media }(>900 €) \\
\text { Muy por encima de la media }(>900 €)\end{array}$ & $\begin{array}{l}9.2 \% \\
22.0 \% \\
48.9 \% \\
20.0 \%\end{array}$ & & \\
\hline
\end{tabular}


En el Anexo 1 se detalla la medición de las variables utilizadas en la presente investigación. Todas las escalas utilizadas en el estudio fueron adaptadas de investigaciones previas y medidas con escalas tipo Likert de siete puntos, desde 1 (totalmente en desacuerdo) hasta 7 (totalmente de acuerdo).

\section{Validación del instrumento de medida}

En el proceso de validación del instrumento de medida se ha tenido en cuenta que el constructo confianza es uno de segundo orden. Por ello, para validar el instrumento de medida se ha procedido en dos etapas, según la recomendación de Ulaga y Eggert (2005).

Se realizó, en primer lugar, un análisis factorial confirmatorio sobre los indicadores de las tres dimensiones de la confianza; tras analizar la fiabilidad y validez convergente y discriminante, dichas dimensiones se convirtieron en variables manifiestas del respectivo constructo de segundo orden mediante una escala sumativa de sus indicadores iniciales. De este modo, el constructo confianza se incorporó al modelo estructural como de primer orden.

En aras de garantizar la validez convergente fueron eliminados aquellos ítemes cuyas cargas factoriales eran no significativas o inferiores a .60 (Bagozzi y Yi, 1988) o para los que el test de los multiplicadores de Lagrange sugería relaciones significativas sobre un factor distinto del que eran indicadores (Hatcher, 1994). Respecto a la fiabilidad, se exigió que todos los $\alpha$ de Cronbach fueran superiores al valor recomendado de .70 (Nunnally y Bernstein, 1994). Se calculó también el índice de fiabilidad compuesta, que debía ser superior también para todos los factores al valor recomendado de .70 (Fornell y Larcker, 1981) y la varianza promedio extraída (AVE), exigiéndose valores superiores a .50 (Fornell y Larcker, 1981) (ver cuadro 2). 


\section{Cuadro 2}

Fiabilidad y validez convergente del concepto confianza

\begin{tabular}{|c|c|c|c|c|c|c|c|}
\hline Dimensión & Indicador & Carga & $\begin{array}{c}\mathrm{t} \\
\text { robusta }\end{array}$ & $\begin{array}{c}\text { Promedio } \\
\text { cargas }\end{array}$ & $\begin{array}{c}\alpha \text { de } \\
\text { Cronbach }\end{array}$ & $\begin{array}{l}\text { Fiabilidad } \\
\text { compuesta }\end{array}$ & AVE \\
\hline \multirow{4}{*}{ Honestidad } & V1 & $0.83^{* *}$ & 16.18 & \multirow{4}{*}{.81} & \multirow{4}{*}{.87} & \multirow{4}{*}{.89} & \multirow{4}{*}{.67} \\
\hline & $\mathrm{V} 2$ & $0.92^{* *}$ & 20.04 & & & & \\
\hline & V3 & $0.89^{* *}$ & 20.19 & & & & \\
\hline & V4 & $0.58^{* *}$ & 9.35 & & & & \\
\hline \multirow{4}{*}{ Benevolencia } & V6 & $0.78^{* *}$ & 16.12 & \multirow{4}{*}{.78} & \multirow{4}{*}{.85} & \multirow{4}{*}{.86} & \multirow{4}{*}{.61} \\
\hline & V7 & $0.85^{* *}$ & 18.97 & & & & \\
\hline & V8 & $0,83^{* *}$ & 18.65 & & & & \\
\hline & V9 & $0,66^{* *}$ & 11.36 & & & & \\
\hline \multirow{5}{*}{ Competencia } & V11 & $0,78^{* *}$ & 16.93 & \multirow{5}{*}{.81} & \multirow{5}{*}{.93} & \multirow{5}{*}{.90} & \multirow{5}{*}{.65} \\
\hline & V12 & $0,81^{* * *}$ & 17.97 & & & & \\
\hline & V13 & $0,83^{* *}$ & 18.49 & & & & \\
\hline & V14 & $0,82^{* *}$ & 15.27 & & & & \\
\hline & V15 & $0,79^{* * *}$ & 15.95 & & & & \\
\hline
\end{tabular}

$\mathrm{S}-\mathrm{B} \chi^{2}(\mathrm{df}=62)=184.17(\mathrm{p}<0.00) ; \mathrm{NFI}=.920 ; \mathrm{NNFI}=.931 ; \mathrm{CFI}=.945 ; \mathrm{IFI}=.946 ; \mathrm{RMSEA}=.081$

$* * \mathrm{p}<.01 ; * \mathrm{p}<.05$

La validez discriminante se evaluó paralelamente bajo dos criterios. En primer lugar, ninguno de los intervalos de confianza al $95 \%$ de las estimaciones de las correlaciones entre cada par de factores contenía el valor 1 (Anderson y Gerbing, 1988). En segundo lugar, la varianza compartida entre cada par de constructos (correlación al cuadrado) era inferior a sus correspondientes índices de varianza extraída (Fornell y Larcker, 1981) (ver cuadro 3).

\section{Cuadro 3}

Validez discriminante del concepto confianza

\begin{tabular}{c|r|r|r}
\hline & \multicolumn{1}{|c|}{$\mathbf{1}$} & \multicolumn{1}{|c}{$\mathbf{2}$} & \multicolumn{1}{|c}{$\mathbf{3}$} \\
\hline 1. Honestidad & $\mathbf{. 6 7}$ & .53 & .66 \\
2. Benevolencia & {$[.65 ; .81]$} & $\mathbf{. 6 1}$ & .57 \\
3. Competencia & {$[.77 ; .88]$} & {$[.68 ; .82]$} & $\mathbf{. 6 5}$ \\
\hline
\end{tabular}

La diagonal representa la varianza extraída promedio AVE. Por encima de la diagonal está la varianza compartida por cada par de factores (correlación al cuadrado). Por debajo de la diagonal el intervalo de confianza al $95 \%$ para la estimación de la correlación entre los factores. 
Una vez constatadas las propiedades psicométricas de la transformación del constructo confianza en factor con indicadores de primer orden, se evaluó si el modelo de medida asociado al modelo estructural gozaba también de las propiedades adecuadas.

Los criterios seguidos para analizar la fiabilidad y validez de dicho modelo de medida fueron los mismos que los aplicados en el proceso de validación anterior. Fue eliminado el ítem V13 del factor norma subjetiva por presentar una carga factorial inferior a 0.6 (Bagozzi y Yi, 1988) y el ítem V17 de la lealtad por sugerir el test de los multiplicadores de Lagrange una relación significativa sobre un factor distinto del que era indicador (Hatcher, 1994). Tras eliminar los mencionados ítemes, los resultados no mostraron problemas de validez convergente (ver cuadro 6); así, el ajuste del modelo razonable (NFI=.88; NNFI=.89; CFI=.91; IFI=.91; RMSEA=.071) y todas las cargas factoriales fueron significativas y superiores a .60. No hubo tampoco problemas de fiabilidad (ver cuadro 4), tanto atendiendo al criterio del $\alpha$ de Cronbach, como del de la fiabilidad compuesta (superior siempre al punto de corte de .70) y la varianza extraída promedio (superior siempre al punto de corte del .50). La validez discriminante del modelo de medida (ver cuadro 5), de acuerdo con ninguno de los dos criterios empleados, presentó problema alguno.

\section{Cuadro 4}

Fiabilidad y validez convergente del modelo final de medida

\begin{tabular}{|c|c|c|c|c|c|c|c|}
\hline Dimensión & Indicador & Carga & $\begin{array}{c}\mathrm{t} \\
\text { robusta }\end{array}$ & $\begin{array}{c}\text { Promedio } \\
\text { cargas }\end{array}$ & $\begin{array}{c}\alpha \text { de } \\
\text { Cronbach }\end{array}$ & $\begin{array}{l}\text { Fiabilidad } \\
\text { compuesta }\end{array}$ & AVE \\
\hline \multirow{3}{*}{ Confianza } & $\mathrm{HON}$ & $.86^{* *}$ & 17.12 & \multirow{3}{*}{.84} & \multirow{3}{*}{.88} & \multirow{3}{*}{.88} & \multirow{3}{*}{.71} \\
\hline & BEN & $.82^{* *}$ & 16.65 & & & & \\
\hline & $\mathrm{COM}$ & $85^{* * *}$ & 21.06 & & & & \\
\hline \multirow{4}{*}{ Actitud } & V4 & $.85^{* *}$ & 14.42 & \multirow{4}{*}{.87} & \multirow{4}{*}{.93} & \multirow{4}{*}{.92} & \multirow{4}{*}{.75} \\
\hline & V5 & $.92^{* *}$ & 16.73 & & & & \\
\hline & V6 & $.95^{* * *}$ & 15.73 & & & & \\
\hline & V7 & $.78^{* *}$ & 12.51 & & & & \\
\hline \multirow{3}{*}{ Control } & V8 & $.91^{* * *}$ & 20.99 & \multirow{3}{*}{.92} & \multirow{3}{*}{.94} & \multirow{3}{*}{.94} & \multirow{3}{*}{.84} \\
\hline & V9 & $.92^{* * *}$ & 21.31 & & & & \\
\hline & V10 & $.93^{* *}$ & 16.69 & & & & \\
\hline \multirow{3}{*}{ Norma } & V11 & $.84^{* * *}$ & 19.36 & \multirow{3}{*}{.86} & \multirow{3}{*}{.90} & \multirow{3}{*}{.90} & \multirow{3}{*}{.75} \\
\hline & V12 & $.86^{* * *}$ & 23.98 & & & & \\
\hline & V14 & $.90^{* * *}$ & 22.99 & & & & \\
\hline \multirow{4}{*}{ Lealtad } & V15 & $.90^{* *}$ & 12.20 & \multirow{4}{*}{.77} & \multirow{4}{*}{.86} & \multirow{4}{*}{.87} & \multirow{4}{*}{.63} \\
\hline & V16 & $.96^{* * *}$ & 15.02 & & & & \\
\hline & V18 & $.62^{* *}$ & 13.33 & & & & \\
\hline & V19 & $.63^{* *}$ & 11.10 & & & & \\
\hline
\end{tabular}

$\mathrm{S}-\mathrm{B} \chi^{2}(\mathrm{df}=104)=267.04(\mathrm{p}<0.00) ; \mathrm{NFI}=.88 ; \mathrm{NNFI}=.89 ; \mathrm{CFI}=.91 ; \mathrm{IFI}=.91 ; \mathrm{RMSEA}=.071$

$* * \mathrm{p}<.01 ; * \mathrm{p}<.05$ 


\section{Cuadro 5}

\section{Validez discriminante del modelo final de medida}

\begin{tabular}{l|r|r|r|r|r}
\hline & \multicolumn{1}{|c|}{$\mathbf{1}$} & \multicolumn{1}{c|}{$\mathbf{2}$} & \multicolumn{1}{c|}{$\mathbf{3}$} & \multicolumn{1}{c|}{$\mathbf{4}$} & \multicolumn{1}{c}{$\mathbf{5}$} \\
\hline 1. Confianza & $\mathbf{. 7 1}$ & .18 & .19 & .12 & .22 \\
2. Actitud & {$[.32 ; .53]$} & $\mathbf{. 7 5}$ & .53 & .53 & .37 \\
3. Control & {$[.33 ; .53]$} & {$[.67 ; .79]$} & $\mathbf{. 8 4}$ & .58 & .40 \\
4. Norma & {$[.23 ; .46]$} & {$[.66 ; .79]$} & {$[.71 ; .82]$} & $\mathbf{. 7 5}$ & .38 \\
5. Lealtad & {$[.37 ; 57]$} & {$[.53 ; .69]$} & {$[.56 ; .71]$} & {$[.53 ; .69]$} & $\mathbf{. 6 3}$ \\
\hline
\end{tabular}

La diagonal representa la varianza extraída promedio AVE. Por encima de la diagonal está la varianza compartida por cada par de factores (correlación al cuadrado). Por debajo de la diagonal el intervalo de confianza al 95\% para la estimación de la correlación entre los factores.

\section{Análisis de resultados}

La estimación del modelo conceptual se realizó mediante el procedimiento de máxima verosimilitud robusto (Satorra y Bentler, 1988), ofreciendo un ajuste razonable (S-B $\chi 2=676.21 ; \mathrm{gl}=113 ; \mathrm{p}=0.000 ; \mathrm{BBNFI}=.902 ; \mathrm{BBNNFI}=.915 ; \mathrm{CFI}=.935$; $\mathrm{IFI}=.936$; RMSEA=.071) (ver cuadro 6).

\section{Cuadro 6}

\section{Contraste de hipótesis}

\begin{tabular}{c|c|l|c|c}
\hline Hipótesis & Signo & \multicolumn{1}{|c|}{ Relación } & $\begin{array}{c}\text { Coeficiente } \\
\text { estandarizado }\end{array}$ & $\begin{array}{c}\text { Valor t } \\
\text { robusto }\end{array}$ \\
\hline $\mathrm{H}_{1}$ & + & Actitud $\rightarrow$ Lealtad & $.281^{* *}$ & 3.88 \\
$\mathrm{H}_{2}$ & + & Control percibido $\rightarrow$ Lealtad & $.335^{* *}$ & 5.98 \\
$\mathrm{H}_{3}$ & + & Norma subjetiva $\rightarrow$ Lealtad & $.240^{* *}$ & 4.87 \\
$\mathrm{H}_{4}$ & + & Confianza $\rightarrow$ Actitud & $.535^{* *}$ & 7.82 \\
$\mathrm{H}_{5}$ & + & Confianza $\rightarrow$ Control percibido & $.538^{* *}$ & 8.56 \\
$\mathrm{H}_{6}$ & + & Confianza $\rightarrow$ Norma Subjetiva & $.468^{* *}$ & 6.71 \\
\hline \multicolumn{5}{c}{$\mathrm{S}-\mathrm{B} \chi^{2}=676.21 ; \mathrm{gl}=113 ; \mathrm{p}=0.000 ;$ BBNFI $=.902 ;$ BBNNFI $=.915 ; \mathrm{CFI}=.935 ; \mathrm{IFI}=.936 ;$} \\
**p $<.01$
\end{tabular}

De los resultados se desprende que todas las variables de la teoría del comportamiento planificado —actitud $\left(\mathrm{H}_{1}: \beta=0.281 ; \mathrm{p}<0.01\right)$, control percibido $\left(\mathrm{H}_{2}\right.$ : $\beta=0.335 ; \mathrm{p}<0.01)$ y norma subjetiva $\left(\mathrm{H}_{3}: \beta=0.240 ; \mathrm{p}<0.01\right)$ - ejercen una influen- 
cia significativa sobre la lealtad. La confianza también desempeña un importante rol en el modelo propuesto, ya que actúa como impulsor de la lealtad de forma indirecta reforzando los efectos positivos de la actitud $\left(\mathrm{H}_{4}: \beta=0.535 ; \mathrm{p}<0.01\right)$, control percibido $\left(\mathrm{H}_{5}: \beta=0.538 ; \mathrm{p}<0.01\right)$ y norma subjetiva $\left(\mathrm{H}_{6}: \beta=0.468 ; \mathrm{p}<0.01\right)$. Este resultado pone de manifiesto que la confianza es un componente relacional importante que ayuda a generar fidelidad online.

\section{Conclusiones}

A pesar de la creciente penetración de Internet en el sector turístico, los factores determinantes de la lealtad hacia el uso de Internet para la compra de productos turísticos todavía no han sido suficientemente analizados desde el punto de vista de la investigación. La facilidad para comparar ofertas y los elevados niveles de incertidumbre a los que se enfrentan los consumidores cuando utilizan canales electrónicos justifican la importancia de estudiar qué factores determinan la lealtad hacia el uso de los servicios turísticos online.

La presente investigación pone de relieve la importancia que adquiere la lealtad en el contexto de la compra online de billetes de avión e identifica cuáles son los principales factores que inciden en su formación. Este trabajo realiza dos contribuciones específicas a la literatura: en primer lugar, presenta un modelo que explica la formación de clientes online leales al establecimiento virtual del operador turístico a través no sólo de las variables del TPB, sino también del papel que desempeña una variable relacional clave como es la confianza; en segundo lugar, contribuye a explicar la lealtad hacia el uso de un tipo de servicio online concreto (los billetes de avión) que ha carecido de la atención debida desde el punto de vista de la investigación.

La revisión de la literatura permite concluir que la lealtad del cliente es esencial para el éxito de un sitio web, por lo que las empresas no deben centrar tanto su objetivo en la atracción sino en la retención de clientes; la confianza es un elemento generador de la relación y motivador de dicha retención. El planteamiento relacional es cada vez más la base de la competitividad de las líneas aéreas, por lo que deben esforzarse en satisfacer a sus clientes y en desarrollar con ellos relaciones a largo plazo (Vázquez et al., 2004).

Los resultados del estudio empírico indican que las variables de la teoría del comportamiento planificado ejercen una influencia directa y significativa en la lealtad 
hacia la compra de billetes de avión. De ese modo, se puede incrementar la lealtad del individuo si, primero, muestra una actitud favorable hacia el uso de Internet como canal de compra; segundo, considera que la tecnología no es compleja y facilita su labor de compra; tercero, recibe opiniones positivas de otros consumidores o de su entorno de referencia. De las tres variables analizadas, es la norma subjetiva la que ejerce una menor influencia sobre la lealtad hacia la compra de billetes de avión. Ello puede ser debido a la experiencia de compra del colectivo analizado (compradores), ya que a medida que se incrementa la experiencia del consumidor éste normalmente evalúa el servicio a partir de sus propias experiencias y no busca tanto las opiniones de terceras personas.

La importancia que adquiere la confianza como antecedente de las creencias de la teoría del comportamiento planificado pone de manifiesto la necesidad de tener en cuenta esta variable en el estudio del comportamiento de compra online. De los resultados se desprende que la confianza es un antecedente clave en la formación de la fidelidad online, puesto que es un componente esencial de las relaciones a largo plazo y de la retención de clientes. Se corrobora en el presente estudio lo ya apuntado en investigaciones previas; esto es, la confianza en el sitio web ejerce un efecto positivo sobre la fidelidad del cliente (Anderson y Srinivasan, 2003; Castañeda, 2005; Flavián y Guinaliu, 2006).

Las conclusiones obtenidas nos permiten plantear un conjunto de implicaciones para la gestión de empresas. La creación de confianza se ha revelado como un factor crucial de cara a que los gestores de las líneas aéreas puedan impulsar el uso de los servicios turísticos online. Dado que la confianza es un constructo multidimensional formado por las dimensiones honestidad, benevolencia y competencia, la línea aérea que desee mejorar los niveles de confianza de sus clientes deberá tener en consideración las diferentes dimensiones que configuran la misma y que influyen en la percepción que sus clientes tienen de su sitio web. En primer lugar, le recomendamos sinceridad y transparencia en cuanto a la posibilidad de cumplimiento de los compromisos y promesas realizadas. En segundo lugar, la política de comunicación de la línea aérea deberá transmitir un mensaje en el que se promueva la consecución de objetivos complementarios a los del consumidor y un mayor bienestar de este último. En tercer lugar, cabría destacar la necesidad de realizar un notable esfuerzo de inversión en aquellos recursos necesarios para la mejora del desempeño en las tareas realizadas y, con ello, favorecer también que los consumidores perciban una mayor competencia y destreza en la organización con la que se relacionan. Por ejemplo, podrían incluir una sección que proporcione información 
general sobre la compañía, su misión y las características generales de la contratación e incorporar centros de atención al cliente que permitan la comunicación con los consumidores a través tanto de canales convencionales como online.

La influencia del control percibido en la lealtad hacia las páginas webs de billetes de avión, sugiere que esta variable es un factor relevante que motiva a la decisión de uso continuo del consumidor de los canales electrónicos. Las webs de venta de billetes de avión que son difíciles de utilizar imponen una mayor barrera cognitiva al usuario que, evidentemente, se refleja en un menor control percibido asociado a su uso. En este sentido, las líneas aéreas deberían tener en cuenta la influencia positiva en la lealtad hacia las mismas de un diseño sencillo y bien estructurado de su web. La relevancia de la actitud como antecedente de la lealtad hacia los canales electrónicos pone de manifiesto la importancia de que los gestores de empresas turísticas analicen de manera continua y sistemática los factores relacionados con el uso del sistema que pueden mejorar dicha actitud, como es la confianza en la línea aérea. Por último, también recomendamos a los gestores que incluyan en la web de venta de billetes de avión la posibilidad de introducir comentarios de otros consumidores o de recomendar la página, ya que la opinión de terceras personas también puede favorecer la lealtad del usuario.

Finalmente, se enumeran las limitaciones del estudio, así como nuevas líneas de investigación de interés futuro. En primer lugar, este estudio se ha aplicado utilizando una muestra de usuarios con experiencia en el servicio considerado. Tal y como postulan Shih y Fang (2004) es necesario complementar las investigaciones para entender las diferencias en la relación entre el control percibido y la decisión de uso entre los consumidores con distintos niveles de experiencia; por tanto, se propone como línea futura el análisis del efecto moderador de la experiencia en el modelo considerado. Otra limitación del estudio es que está restringido al uso de un servicio específico (billetes de avión); por este motivo, se propone contrastar el modelo con una muestra de compradores de otro tipo de servicios turísticos y comparar los resultados obtenidos. Por último, las compras de productos turísticos a distancia incluyen distintas formas de tecnología, pudiendo variar el comportamiento del consumidor en función del canal utilizado; por consiguiente, se propone como futura línea de investigación aplicar el modelo propuesto a una muestra de compradores de billetes de avión a través del móvil con el fin de comparar los resultados obtenidos. 


\section{Referencias}

AIMC (2007). Navegantes en la red. Disponible en http://wwwaimc.es

Ajzen, I. (1985). From intentions to actions; a theory of planned behaviour. Actioncontrol: from cognition to behaviour. J. Kuhi, y J. Beckmann, eds. Springer: Heilderberg: 11-39.

(1991). The Theory of Planned Behavior. Organizational Behavior and Human Decision Processes 50 (2): 179-211.

y T.J. Madden (1986). Prediction of goal-directed behavior: attitudes, intentions and perceived behavioral control. Journal of Experimental Social Psychology 22: 453-474.

Anderson, J.C. y D.W. Gerbing (1988). Structural equation modelling in practice: A review and recomended two-step approach. Psychological Bulletin 103: 411-423.

Anderson, R. y S. Srinivasan (2003). E-Satisfaction and E-Loyalty: A Contingency Framework. Psychology \& Marketing 20 (2): 123-138.

Bagozzi, R.P. y Y. Yi (1988). On the evaluation of structural equation models. Journal of the Academy of Marketing Science 16 (1): 74-94.

Barroso, C. y E. Martín (1999). Nivel de servicio y retención de clientes: el caso de la banca en España. Revista Española de Investigación de Marketing ESIC, marzo 4: 9-36.

Bhattacherjee, A. (2002). Individual trust in online firm: scale development initial test. Journal of Management Information Systems 19 (1): 211-241.

Blau, P.M. (1964). Exchange and Power in Social Life, New York: Wiley.

Castañeda, J.A. (2005). La fidelidad en Internet, Marketing en Internet. A.M. Gutiérrez y M.J. Sánchez-Franco, eds. Madrid: Pirámide: 237-276. 
Chai, L. y P. Pavlou (2002). Customer relationship Management.com: a Cross-cultural empirical Investigation of electronic Commerce. Americas Conference on Information Systems (AMCIS) Proceedings, Association for Information Systems, ed.: 483-491. Disponible en: http://aisel.aisnet.org/amcis2002/70.

Chang, C. L-H. (2007). The political behavior intention of user in information system development. Human Systems Management 26: 123-137.

Dick, A. y K. Basu (1994). Customer loyalty: toward an integrated conceptual framework. Journal of the Academy of Marketing Science 22 (2): 99-113.

Doney, P.M. y Cannon, J.P. (1997). An examination of the nature of trust in buyerseller relationships. Journal of Marketing, abril 61: 35-51.

East, R. (1993). Investment decisions and the theory of planned behaviour. Journal of Economic Psychology 14: 337-375.

Engel, J., D. Kollat y R. Blackwell (1982). Consumer behavior. 4a. ed. Hynsdale: The Dryden Press.

Fernández, M. y J.D. Martín (2006). La confianza y el compromiso como factores clave del éxito de las relaciones comerciales: una aplicación empírica en el sistema de franquicia. Revista Europea de Dirección y Economía de la Empresa 15 (1): 77-100.

Fishbein, M. e I. Ajzen (1975). Belief, attitude, intention and behavior: an introduction to theory and research, New York: Addison-Wesley.

Flavián, C. y M. Guinalíu (2006). Consumer trust, perceived security, and privacy policy: three basic elements of loyalty to a web site. Industrial Management \& Data Systems 106 (5/6): 601-620.

(2007). Un análisis de la influencia de la confianza y del riesgo percibido sobre la lealtad a un sitio web. El caso de la distribución de servicios gratuitos. Revista Europea de Dirección y Economía de la Empresa 16 (1): 159-178. 
Fornell, C. y D.F. Larcker (1981). Evaluating structural equations models with unobservable variables and measurement error. Journal of Marketing Research 18: 39-50.

Ganesan, S. (1994). Determinants of long-term orientation in buyer-seller relationships. Journal of Marketing, abril 58: 1-19.

George, J.F. (2004). The theory of planned behavior and Internet purchasing. Internet Research 14 (3): 198-212.

George, M.Z. (2002). Promoting services via the Internet: new opportunities and challenges. Journal of Services Marketing 16 (5): 412-423.

Gilbert, D., J. Powell-Perry y S. Widijoso (1999). Approaches by hotels to the use of the internet as a relationship marketing tool. Journal of Marketing Practice: Applied Marketing Science 5 (1): 21-38.

Hair, J., R. Anderson, R. Tatham y W. Black (1999). Análisis Multivariante, Madrid: Pearson Educacion.

Hatcher, L. (1994). A step by step approach to using the SAS system for factor analysis and structural equation modeling, SAS Institute Inc: Cary.

Jarvenpaa, S., N. Tractinsky y M. Vitale (2000). Consumer trust in an Internet store. Information Technology and Management 1 (1/2): 45-71.

Keller, K. (1993). Conceptualizing, measuring and managing customer-based brand equity. Journal of Marketing 57: 1-22.

Kim, H., T. Kim y S.W. Shim (2009). Modelling roles of subjective norms and eTrust in customers' acceptance of airline B2C ecommerce websites. Tourism Management 30 (2): 266-277.

Lee, C. y R.T. Green (1991). Cross-cultural examination of the Fishbein behavioral intentions model. Journal of International Business Studies 21 (2): 289-305.

Lee, R., J. Murphy y L. Neale (2009). The interactions of consumption characteristics on social norms. Journal of Consumer Marketing 26 (4): 277-285. 
Lobb, A.E., M. Mazzocchi y W.B. Traill (2007). Modelling risk perception and trust in food safety information within the theory of planned behaviour. Food Quality and Preference 18: 384-395.

Mcknight, D.H., L.L. Cummings y N.L. Chervany (1998). Initial trust formation in new organizational relationships. Academy of Management Review 23 (3): 473-490.

Morgan, R. y S. Hunt (1994). The commitment trust theory of relationship marketing. Journal of Marketing, julio 58: 20-38.

Nadiri, H., K. Hussain, E.H. Ekiz y S. Erdogan (2008). An investigation on the factors influencing passengers' loyalty in the North Cyprus national airline. The TQM Journal 20 (3): 265-280.

Nunnally, J.C. e I.H. Bernstein (1994). Psychometric Theory. 3a. ed., New York: McGraw-Hill.

Pavlou, P. y L. Chai (2002). What drives electronic commerce across cultures? A cross-cultural empirical investigation of the theory of Planned behaviour. Journal of Electronic Commerce Research 3 (4): 240-253.

Pavlou, P.A. (2003). Consumer Intentions to Adopt Electronic Commerce-Incorporating Trust and Risk in the Technology Acceptance Model, International Journal of Electronic Commerce 7 (3): 101-123.

y M. Fygenson (2005). Understanding and Predicting Electronic Commerce Adoption: An Extension of the Theory of Planned Behavior. MIS Quarterly 30 (1): 115-144.

Peterson, R., S. Balasubramanian y B. Bronnenberg (1997). The exploring the implications on the Internet for the consumer marketing. Journal of the Academy Marketing Science 25 (4): 329-346.

Red.es (2009). Estudio sobre Comercio Electrónico B2C 2009. Disponible en http://www.red.es/media/2008-10/1224586013979.pdf. 
Roy, M., O. Dewit y B. Aubert (2001). The impact of interface usability on trust in web retailers. Internet Research: Electronic Networking Applications and Policy 11 (5): 388-398.

San Martín, S. (2006). La generación de confianza del consumidor en el establecimiento comercial. Una perspectiva multinivel. Revista Europea de Dirección y Economía de la Empresa 15 (1): 201-224.

_ J. Gutiérrez y M.J. Camarero (2004). Dimensiones y determinantes del compromiso relacional del consumidor. Revista Española de Investigación de Marketing Esic 8 (1): 97-125.

Satorra, A. y P.M. Bentler (1988) Scaling corrections for chi square statistics in covariance structure analysis, American Statistical Associations Proceedings of the Business and Economic Sections, American Statistical Association, Alexandria, VA: 308-313.

Schifter, D.B. e I. Ajzen (1985). Intention, perceived control and weight loss: an application of the theory of planned behaviour. Journal of Personality and Social Psychology 49: 842-851.

Schofield, J.W. (1974). Effect of norms, public disclosure and ned for approval on volunteering behavior consistent with attitudes. Journal of Personality and Social Psychology 31: 1126-1133.

Shankar, V., A. Smith y A. Rangaswamy (2003). Customer satisfaction and loyalty in online and offline environments. International Journal of Research in Marketing 20: 153-175.

Shih, Y. y K. Fang (2004). The use of a decomposed theory of planned behaviour to study Internet banking in Taiwan. Internet Research 14 (3): 213-223.

Steenkamp, J. y H. Baumgartner (2000). On the use of structural equation models for marketing modelling. International Journal of Research in Marketing 17 (2/3): 195-202.

Suh, B. e I. Han (2002). Effect of trust on customer acceptance of Internet banking. Electronic Commerce Research and Applications 1 (3/4): 247-263. 
Taylor, S. y P. Todd (1995). Understanding information technology usage: a test of competing models. Information Systems Research 6 (2): 144-176.

Tsai, T. (2006) An extension of TAM with TPB for online game to predict loyalty, Master Thesis. Disponible en http://203.64.120.207/ETD-db/ETD-search/ view_etd?URN=etd-0801107-104226.

Ulaga, W. y A. Eggert (2005). Relationship value in business markets: the construct and its dimensions. Journal of Business-to-Business Marketing 12 (1): 73-99.

Vázquez, R., A.M. Díaz y A. Suárez (2004). Cómo usan Internet las líneas aéreas para desarrollar relaciones estables con los clientes. Universia Business Review 002: 34-47.

Wu, I-L. y J-L. Chen (2005). An extension of trust and TAM model with TPB in the initial adoption of on-line tax: An empirical study. International Journal of Human-Computer Studies 62: 784-808.

Zeithaml, V.A., L.L. Berry y A. Parasuraman (1996). The behavioural consequences of service quality. Journal of Marketing 60 (2): 31-46.

Zineldin, M. (2000). Beyond relationship marketing: technologicalship marketing. Marketing Intelligence \& Planning 18 (1): 9-23. 


\section{Anexo 1}

\begin{tabular}{|c|c|c|c|}
\hline & Constructo & Descripción & Fuente \\
\hline \multirow{15}{*}{ 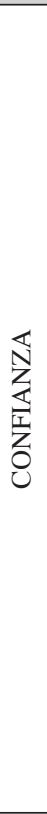 } & \multirow{5}{*}{ Honestidad } & $\begin{array}{l}\text { Creo que este tipo de webs suelen cumplir los } \\
\text { compromisos que asumen }\end{array}$ & \multirow{15}{*}{$\begin{array}{c}\text { Adaptado de: } \\
\text { Doney y Canon (1997), } \\
\text { Roy et al. (2001), } \\
\text { Flavián y Guinaliu (2006). }\end{array}$} \\
\hline & & Creo que la información que ofrecen es veraz y honesta & \\
\hline & & Creo que puedo fiarme de las condiciones que ofrecen & \\
\hline & & Nunca realizan falsas afirmaciones & \\
\hline & & $\begin{array}{l}\text { Se caracterizan por su transparencia al ofrecer sus } \\
\text { servicios al usuario }\end{array}$ & \\
\hline & \multirow{6}{*}{ Benevolencia } & $\begin{array}{l}\text { Creo que los consejos y recomendaciones que estas } \\
\text { webs ofrecen al usuario buscan un beneficio mutuo. }\end{array}$ & \\
\hline & & $\begin{array}{l}\text { Creo que se preocupan por los intereses/beneficios } \\
\text { presentes y futuros de sus usuarios. }\end{array}$ & \\
\hline & & $\begin{array}{l}\text { Creo que tienen en cuenta las repercusiones que sus } \\
\text { acciones pueden tener sobre sus usuarios. }\end{array}$ & \\
\hline & & $\begin{array}{l}\text { Creo que no harían nada que pudiera perjudicar a sus } \\
\text { usuarios de forma intencionada. }\end{array}$ & \\
\hline & & $\begin{array}{l}\text { Creo que al diseñar su oferta comercial tienen en cuenta } \\
\text { los deseos y necesidades de los usuarios. }\end{array}$ & \\
\hline & & Creo que atienden las necesidades de sus usuarios. & \\
\hline & \multirow{4}{*}{ Competencia } & $\begin{array}{l}\text { Creo que tienen la capacidad necesaria para realizar su } \\
\text { trabajo. }\end{array}$ & \\
\hline & & $\begin{array}{l}\text { Creo que tienen suficiente experiencia en la } \\
\text { comercialización de los productos/servicios que } \\
\text { ofrecen. }\end{array}$ & \\
\hline & & $\begin{array}{l}\text { Creo que tienen los recursos necesarios para realizar } \\
\text { con éxito sus actividades. }\end{array}$ & \\
\hline & & $\begin{array}{l}\text { Creo que conocen suficientemente a los usuarios como } \\
\text { para ofrecerles productos/servicios adaptados a sus } \\
\text { necesidades. }\end{array}$ & \\
\hline \multirow{11}{*}{$\stackrel{n}{\hat{F}}$} & \multirow{4}{*}{ Actitud } & Es una idea que me gusta. & \multirow{4}{*}{$\begin{array}{c}\text { Adaptado de: } \\
\text { Taylor y Todd (1995), } \\
\text { Bhattacherjee (2002), } \\
\text { George (2004). }\end{array}$} \\
\hline & & Me parece una idea inteligente. & \\
\hline & & Es una buena idea. & \\
\hline & & Me parece una experiencia positiva. & \\
\hline & & $\begin{array}{l}\text { Utilizar Internet para comprar billetes de avión es algo } \\
\text { que tengo controlado. }\end{array}$ & Adaptado de: \\
\hline & Control percibido & $\begin{array}{l}\text { Tengo los recursos, el conocimiento y la habilidad para } \\
\text { utilizar Internet para comprar billetes de avión. }\end{array}$ & $\begin{array}{l}\text { Taylor y Todd (1995), } \\
\text { Bhattacherjee (2002), }\end{array}$ \\
\hline & & $\begin{array}{l}\text { Soy capaz de utilizar Internet para comprar billetes de } \\
\text { avión. }\end{array}$ & George (2004). \\
\hline & & $\begin{array}{l}\text { La gente cuyas opiniones valoro aprueban que utilice } \\
\text { Internet para comprar billetes de avión. }\end{array}$ & \\
\hline & Norma & $\begin{array}{l}\text { La mayoría de personas a las que tengo en cuenta } \\
\text { piensan que debo utilizar Internet para comprar billetes } \\
\text { de avión. }\end{array}$ & $\begin{array}{l}\text { Adaptado de: } \\
\text { Taylor y Todd (1995), } \\
\text { Bhattacherjee (2002), }\end{array}$ \\
\hline & subjetiva & $\begin{array}{l}\text { Se espera de mí que utilice Internet para comprar } \\
\text { billetes de avión. }\end{array}$ & George (2004). \\
\hline & & $\begin{array}{l}\text { Las personas cercanas a mi están de acuerdo con que } \\
\text { utilice Internet para comprar billetes de avión. }\end{array}$ & \\
\hline & & $\begin{array}{l}\text { Intento utilizar cada vez más Internet para comprar } \\
\text { billetes de avión. }\end{array}$ & \\
\hline$\frac{1}{4}$ & & $\begin{array}{l}\text { Cuando necesito comprar billetes de avión, Internet es } \\
\text { mi primera opción. }\end{array}$ & \\
\hline 点 & & $\begin{array}{l}\text { Hago comentarios positivos de la compra de billetes de } \\
\text { avión online a otras personas. }\end{array}$ & $\begin{array}{l}\text { Adaptado de: } \\
\text { Zeithaml et al. (1996) }\end{array}$ \\
\hline 可 & & $\begin{array}{l}\text { Recomiendo el uso de Internet para comprar billetes de } \\
\text { avión a cualquiera que me pida consejo. }\end{array}$ & \\
\hline & & $\begin{array}{l}\text { Animo a amigos y familiares a que utilicen los servicios } \\
\text { de Internet para comprar billetes de avión. }\end{array}$ & \\
\hline
\end{tabular}

